\title{
Self-Limiting Polymerization of DNA Origami Subunits with Strain Accumulation
}

Jonathan F. Berengut ${ }^{1}$, Chak Kui Wong ${ }^{2}$, Julian C. Berengut ${ }^{3}$, Jonathan P. K. Doye ${ }^{2}$, Thomas E. Ouldridge $e^{4}$ Lawrence K. Lee $e^{l *}$

${ }^{1}$ EMBL Australia Node for Single Molecule Science, School of Medical Sciences, UNSW Sydney, 2052, Australia.

${ }^{2}$ Physical \& Theoretical Chemistry Laboratory, Department of Chemistry, University of Oxford, South Parks Road, Oxford, OX1 3QZ, United Kingdom.

${ }^{3}$ School of Physics, UNSW Sydney, 2052, Australia.

${ }^{4}$ Department of Bioengineering and Centre for Synthetic Biology, Imperial College London, London, SW7 2AZ, United Kingdom.

*Correspondence to: lawrence.lee@unsw.edu.au

\section{ABSTRACT:}

Biology demonstrates how a near infinite array of complex systems and structures at many scales can originate from the self-assembly of component parts on the nanoscale. But to fully exploit the 
benefits of self-assembly for nanotechnology, a crucial challenge remains: How do we rationally encode well-defined global architectures in subunits that are much smaller than their assemblies? Strain accumulation via geometric frustration is one mechanism that has been used to explain the self-assembly of global architectures in diverse and complex systems a posteriori. Here we take the next step and use strain accumulation as a rational design principle to control the length distributions of self-assembling polymers. We use the DNA origami method to design and synthesise a molecular subunit known as the PolyBrick, which perturbs its shape in response to local interactions via flexible allosteric blocking domains. These perturbations accumulate at the ends of polymers during growth, until the deformation becomes incompatible with further extension. We demonstrate that the key thermodynamic factors for controlling length distributions are the intersubunit binding free energy and the fundamental strain free energy, both which can be rationally encoded in a PolyBrick subunit. While passive polymerisation yields geometrical distributions, which have the highest statistical length uncertainty for a given mean, the PolyBrick yields polymers that approach Gaussian length distributions whose variance is entirely determined by the strain free energy. We also show how strain accumulation can in principle yield length distributions that become tighter with increasing subunit affinity and approach distributions with uniform polymer lengths. Finally, coarse-grained molecular dynamics and Monte Carlo simulations delineate and quantify the dominant forces influencing strain accumulation in a molecular system. This study constitutes a fundamental investigation of the use of strain accumulation as a rational design principle in molecular self-assembly.

KEYWORDS: DNA nanotechnology, polymer thermodynamics, geometrically frustrated assemblies, self-assembly, self-limiting polymer, multi-subunit assembly, molecular dynamics 
DNA nanotechnology utilizes the well-known Watson and Crick base-pairing rules to facilitate the programmed self-assembly of nanoscale structures with specified geometries. ${ }^{1-3}$ The DNA origami method is particularly useful for nanoscale construction because it allows for the highyield synthesis of complex $2 \mathrm{D}$ and $3 \mathrm{D}$ structures. ${ }^{4-10}$ Long single-stranded DNA (ssDNA) scaffolds are 'folded' into the desired structure in the presence of an excess of short ssDNA 'staple' oligonucleotides, which hybridize to specific regions of the scaffold. The molar excess of short DNA staples ensures that the maximally-hybridized target structure is thermodynamically favored. ${ }^{4}$ Moreover, since each structure consists of a single DNA scaffold strand, every location on a DNA origami structure is uniquely addressable, and can be specifically functionalized to control the spatial localization of molecules or nanoparticles with high precision $(<10 \mathrm{~nm}$ resolution). ${ }^{11-14}$ DNA origami thus provides enormous dexterity with nanoscale engineering including for the construction of nanoscale machines, ${ }^{15-19}$ sensors, ${ }^{20-24}$ nanoscale calibration ${ }^{25-29}$ as well as for the exploration of fundamental questions in biology and molecular self-assembly. ${ }^{30-33}$

The size of DNA origami structures is however limited by the length of the scaffold strand, which typically consists of a ssDNA bacteriophage genome, such as that of the 7249-nucleotide (nt) M13mp18 phage. ${ }^{4}$ Thus, an important challenge in DNA nanotechnology is the construction of well-defined structures at larger scale. One way to construct larger DNA origami structures is by synthesizing larger ssDNA scaffolds. Such 'super-sized' DNA origami structures have been synthesized from $26 \mathrm{knt}^{34}$ and $51 \mathrm{knt}^{35}$ scaffolds. However, longer DNA scaffold strands require a proportionally greater number of unique staple strands, thus leading to higher synthesis cost. Moreover, it remains unclear how synthesis yields are affected by scaffold length, particularly in 3D structures, which likely present larger kinetic barriers to assembly. An alternative approach is the hierarchical self-assembly of uniform and well-defined structures from multiple DNA origami 
subunits. Multi-subunit structures offer the opportunity to incorporate greater complexity and to bridge the gap between nanoscale and macroscale engineering. ${ }^{36-38}$

Assembly of multi-subunit DNA origami structures can be directed via sequence-specific hybridization of complementary unpaired DNA strands at specific sites on interacting subunits. ${ }^{4-5}$, ${ }^{39}$ Alternatively, shape-specific DNA base-stacking can be used by arranging DNA blunt-ends on one subunit with shape complementarity to DNA blunt ends in a binding partner. ${ }^{17,36,40}$ Binding energies for both mechanisms can be controlled by the number, shape and sequence of binding sites. ${ }^{17,41}$ Homologous DNA origami subunits that bind to each other can spontaneously form linear, 2D or 3D lattices with well-defined internal geometry. However without mechanisms aside from the depletion of subunits to terminate growth, such 'passive' polymerization results in illdefined global dimensions (Figure 1A)..$^{42-48}$

Construction of multi-subunit superstructures with well-defined global dimensions presents the additional challenge of controlling the termination of assembly. One approach involves the use of heterogeneous subunits, which self-terminate because each subunit within the assembled superstructure is distinct and has a specific location within the assembled superstructure (Figure 1B). $5,17,37,40,45,49-51$ Consequently, each subunit in heterologous superstructures is uniquely addressable. ${ }^{40}$ However, since larger structures require a greater number of distinct subunits, they are more expensive, both in raw materials and synthesis complexity. Yield of heterologous superstructures decreases exponentially with subunit count ${ }^{37}$ because of the increasing number of possible undesirable interactions that can trap the synthesis pathway in malformed assemblies. Yields can be improved by the hierarchical addition of different subunits during assembly and by optimizing their stoichiometric ratio. Termination can also be controlled with external mechanisms that confine growth. For example, separate structures or templates can be used to reduce binding 
energies for otherwise unfavorable intermolecular interactions. Growth does not extend beyond the template and thus terminates spontaneously at its edge (Figure 1C). ${ }^{52-53}$ The problem of defining global dimensions is therefore shifted from the design of the subunits to the synthesis of the template. Importantly, the use of templates to control termination is distinct from the use of templates to control nucleation and overcome kinetic barriers. ${ }^{54-55}$ Termination of nucleated polymers can also be controlled temporally by the addition of capping structures at fixed time points to block further growth (Figure 1D). ${ }^{56}$ Vernier assembly combines heterologous subunits with templating to terminate polymerization (Figure 1E). Subunits differ in length and have a proportionally different number of complementary binding sites, allowing heterologous subunits to bind with different offset distances. When growth reaches a length corresponding to a common multiple of the number of binding sites, subunits come into register with all binding sites occupied, thus resulting in self-termination. ${ }^{57-58}$ As above however, maximizing yield with heterologous super structures requires a complex balance between binding affinities, kinetics, environmental conditions and stoichiometric ratios..$^{57-58}$

Alternative mechanisms where the global superstructure is entirely encoded in multiple identical subunits are therefore interesting and desirable. Several mechanisms for such self-limiting assembly at equilibrium have been explored. ${ }^{59}$ These include self-closing assemblies where subunits bind with a preferred orientation yielding closed and symmetrical superstructures such as rings or polyhedra, the global dimensions of which can be encoded in the shape of subunits. ${ }^{4-5,36 \text {, }}$ ${ }^{39}$ For example, subunits with angled binding sites will naturally self-assemble into closed rings where all binding sites are occupied (Figure 1F). ${ }^{36}$ The size and number of subunits in the ring depends on the angle, which is tightly controlled for superstructures whose global dimensions are shorter than their persistence length but is increasingly less well defined for larger structures. ${ }^{36}$ In 
one manifestation, the angle of binding sites in subunits was reconfigurable to control the distribution of ring sizes. ${ }^{60}$ Self-closing assemblies are common in biology such as in the formation of ring structures in the bacterial flagellar motor ${ }^{61-62}$ or the closed spherical shells of viral capsids ${ }^{63}$ and the formation of bilayers or micelles from amphiphilic phospholipids. ${ }^{64}$

Self-termination in identical subunits that do not form closed structures is perhaps a more difficult task. Unlike the mechanisms for self-termination presented above, there are neither external confinement mechanisms nor does a fully assembled structure have all its binding sites occupied. Thus, self-termination in open structures must utilize fundamentally different mechanisms. ${ }^{59}$ Uchida et al. approached the problem by designing a subunit that encompassed a rotary Vernier mechanism to control the mean length of polymers ${ }^{65}$ Another proposed mechanism entails the accumulation of strain as subunits are added to a growing polymer. ${ }^{66-69}$ Physically, strain accumulation can be achieved by a mechanism known as geometric frustration where local interactions yield global geometries that are unfavorable to continuous uniform propagation (Figure 1G,H). Geometric frustration has been implicated in a diverse range of assemblies including chiral protein filaments, ${ }^{70}$ molecular crystals ${ }^{71}$ and membranes,${ }^{72}$ the formation of vertices in viral capsids ${ }^{73}$ and the crystalline coating of droplets with nanoparticles. ${ }^{74}$ However, there are many open questions on the resulting structure, thermodynamics and kinetics of geometrically frustrated assemblies and there remains a major challenge of its use as a rational design principle..$^{75}$

Here, we rationally design and construct a synthetic system that utilizes strain accumulation via geometric frustration to control the length distribution of one-dimensional polymers that selfassemble from identical DNA origami subunits. 
We present a thermodynamic model which explains how strain accumulation can be an effective means to control self-termination during assembly. The model predicts how the rate of strain accumulation versus polymer length quantifiably alters the mean, variance and shape of polymer length distributions. We then present a DNA origami structure, which we call the PolyBrick, to embody a molecular subunit capable of strain accumulation via an allosteric displacement mechanism proposed by Penrose in $1959 .{ }^{76}$ We characterize the structure and dynamics of the PolyBrick with electron microscopy and coarse-grained molecular dynamics simulations using the oxDNA model. ${ }^{77}$ Finally, we utilize the PolyBrick to demonstrate control over self-termination of linear polymerization with strain accumulation. Moreover, by tuning the extent of the allosteric effect, the mean size of the superstructure can be encoded into each individual subunit as well as the variance, which is dramatically lower for PolyBricks compared with passive uncontrolled DNA origami polymers. 

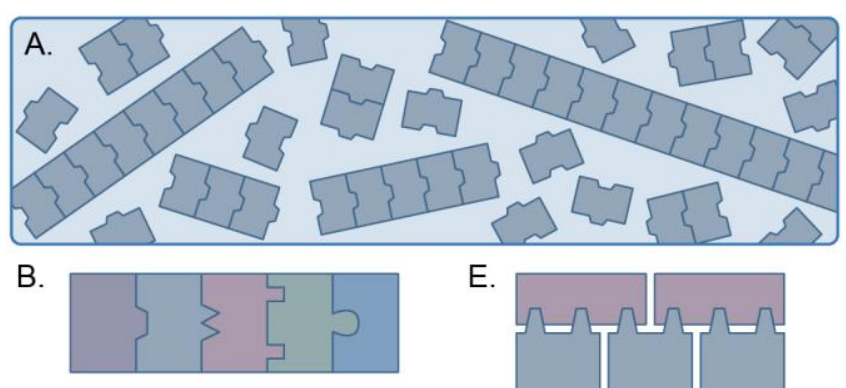

E.

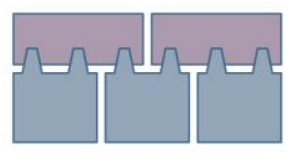

C.

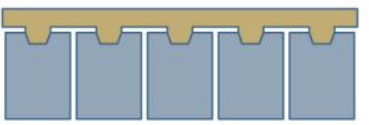

F.

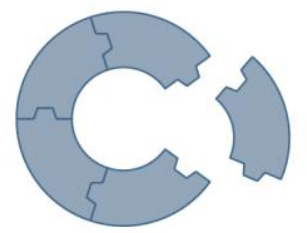

D.
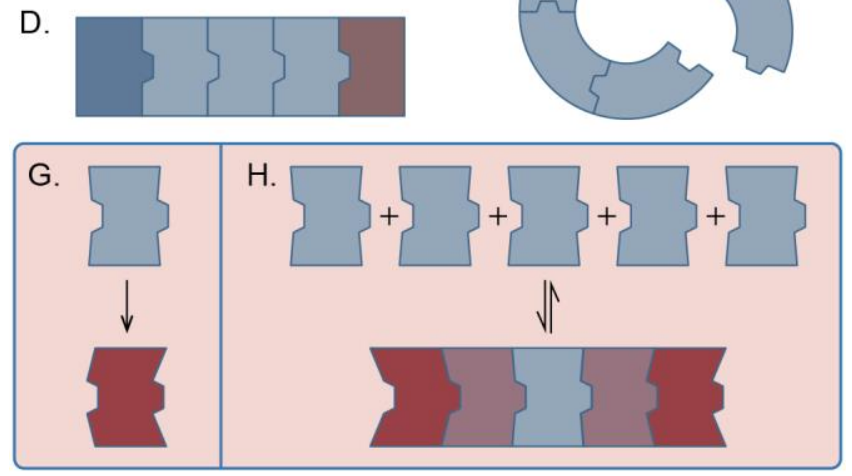

Figure 1. Methods to control the termination of linear polymerization. (A) Identical subunits with complementary binding sites interact passively to form polymers with a wide distribution of lengths. (B) A self-terminating superstructure composed of five heterogeneous subunits. (C) A separate structure can be used to template the assembly of five subunits into a superstructure. (D) Capping structures may be introduced to terminate polymerization temporally. (E) A Vernier assembly comprising two 3-site subunits and three 2-site subunits. (F) Self-termination of superstructure assembly by ring closure occurs when binding sites on each subunit are oriented at an angle. (G) A strain accumulation subunit with allosteric binding sites, which can deform when force is applied. (H) Five identical strain accumulation subunits which form a geometrically frustrated polymer. 


\section{RESULTS}

Thermodynamic model for polymerisation with strain accumulation.

To understand the thermodynamics of linear polymerisation with strain accumulation, we consider a reaction that describes the formation of a polymer of length $n\left(S_{n}\right)$, by the addition of the $n t h$ monomeric subunit $(S)$ to an existing polymer:

$$
S+S_{n-1} \rightleftharpoons S_{n}
$$

Polymerisation with strain accumulation requires that there is a free energy cost for the addition of subunits to a polymer that increases with length. Thus, the standard state free energy associated with this polymer extension reaction can be defined as:

$$
\Delta G_{\mathrm{ext}}^{0}(n)=\Delta G_{b}^{0}+\Delta G_{s}^{0}(n)
$$

$\Delta G_{b}^{0}$ is the standard free energy of binding between subunits and $\Delta G_{s}^{0}(n)$ quantifies the positive strain free energy for the addition of a single subunit, which increases with polymer length (Figure 2A). Assuming a large number of molecules, $\Delta G_{\text {ext }}^{0}(n)$ is related to the equilibrium molar concentrations of subunits, $[\mathrm{S}]$ :

$$
\Delta G_{\mathrm{ext}}^{0}(n)=-R T \ln \left(\frac{\left[S_{n}\right]}{[S]\left[S_{n-1}\right]}\right)
$$

where $R$ is the Boltzmann constant in units of $\mathrm{kJ} / \mathrm{mol}$ and $T$ is the temperature in Kelvin. Combining equations (2) and (3), we can express the equilibrium concentration of polymers as:

$$
\left[S_{n}\right]=[S]\left[S_{n-1}\right] e^{\frac{-\left(\Delta G_{b}^{0}+\Delta G_{S}^{0}(n)\right)}{R T}}
$$


Given a known total concentration of subunits in the system, $\left[S_{\text {total }}\right]$, the coupled simultaneous equations of (4) can be solved along with

$$
\left[S_{\text {total }}\right]=\sum_{i=1}^{n} i\left[S_{i}\right],
$$

which ensures conservation of molecular units by summing over polymers of all lengths. To understand the effects of strain accumulation on polymer length distributions, it is useful to quantify the equilibrium concentration of polymers of length $n$ relative to monomers according to

$$
\left[S_{n}\right]=[S]^{n} e^{\frac{-\Delta G_{P}^{0}(n)}{R T}},
$$

where $\Delta G_{p}^{0}(n)$ is the total standard free energy associated with the formation of the polymer of length $n$.

In the absence of strain accumulation, $\Delta G_{p}^{0}(n)=(n-1) \Delta G_{b}^{0}$ and equation (6) describes the polymerisation of passive subunits (Figure $2 \mathrm{~B}, \mathrm{C}$ ). This assumption yields a geometric probability distribution of lengths where monomers are the most abundant species and the probability of a randomly-chosen polymer having length $n, P\left(\left[S_{n}\right]\right)$, decreases exponentially (Figure 2D). Furthermore, given $\left[S_{\text {total }}\right]$, the mean polymer length $(\bar{n})$ and the variance $\left(\sigma^{2}\right)$ can be calculated analytically for a given binding affinity $\left(\Delta G_{b}^{0}\right)$ (Supplementary Note 1$) \cdot \bar{n}$ increases with affinity as expected and the variance of a geometric distribution is large and well approximated as the square of the mean for large means (Figure 2E). Indeed, the geometric distribution has the highest statistical entropy of all distributions supported on positive integers with a given mean. Thus, passive polymerisation affords the least possible control of polymer length distributions (Figure 2F). 
Now we consider a subunit that accumulates strain during polymerisation such as a version of a displacement accumulation model proposed by Penrose in $1959^{76}$ (Figure 2G). In this model, the binding of subunits physically displaces a regulatory domain by a distance that increases with polymer length up to a maximal distance set by the physical dimensions of the subunit. With inbuilt resistance to the displacement of the regulatory domain from a 'resting' position, strain also accumulates prior to reaching the maximal displacement distance (Figure $2 \mathrm{H}$ ). The rate of strain accumulation depends on the incremental increase of strain free energy associated with extension by a subunit. A linear increment in strain free energy contribution for incorporation of each additional subunit (Figure 2I) can be given as:

$$
\Delta G_{s}^{0}(n)=(n-1) \Delta G_{s, \mathrm{dim}}^{0}
$$

where the increment, $\Delta G_{s, \text { dim }}^{0}$, is defined as the positive strain free energy associated with the formation of a dimer. With equation (7) we can see that the standard free energy associated with polymer formation is then given by:

$$
\Delta G_{p}^{0}(n)=\sum_{i=1}^{n-1}\left(\Delta G_{b}^{0}+i \Delta G_{s, \mathrm{dim}}^{0}\right)=(n-1) \Delta G_{b}^{0}+\left(\frac{n^{2}-n}{2}\right) \Delta G_{s, \mathrm{dim}}^{0}
$$

Thus, a linear increment in strain free energy results in a total polymer strain free energy that accumulates quadratically. By substituting equation (8) into equation (6) we see that $P\left(\left[S_{n}\right]\right)$ is an exponential of a quadratic. Consequently, for sufficiently large average polymer size, when the polymer length can be approximated as continuous, $P\left(\left[S_{n}\right]\right)$ is approximately Gaussian (Figure $2 \mathrm{~J}, \mathrm{~K}$ and Supplementary Note 2). Moreover, in this limit, the variance is constant $\left(\sigma^{2}=\frac{R T}{\Delta G_{s, \mathrm{dim}}^{0}}\right)$ and independent of $\Delta G_{b}^{0}$ and $\left[S_{\text {total }}\right]$ (Figure $2 \mathrm{~L}$ ). The mean cannot be expressed analytically but can be determined numerically and depends on $\Delta G_{b}^{0}, \Delta G_{s, \text { dim }}^{0}$ and $\left[S_{\text {total }}\right]$ (Figure $2 \mathrm{M}$ ). The 
underlying physical mechanism can also be arrived at intuitively: by limiting the formation of long polymers, strain accumulation frees a substantial proportion of subunits that would otherwise be incorporated into longer polymers. Moreover, the strain free energy will have a relatively small destabilizing effect on the probability of forming shorter polymers. Consequently, the additional free subunits will come together to form shorter polymers and the peak of the equilibrium length distribution shifts away from monomers towards a finite mean polymer length. Thus, strain accumulation can yield symmetrical length distributions that differ fundamentally from those of passive linear polymers with a dramatically lower variance that in principle can be independent of polymer length.

The system of equations above can be generalised to allow consideration of other non-linear strain models. For example, if an ideal spring resists displacement (Figure 2N), the force associated with displacement follows Hooke's law where $\Delta G_{s, \mathrm{dim}}^{0}$ is similar to the spring constant and the increment in strain free energy therefore increases quadratically (Figure 2O) according to:

$$
\Delta G_{s}^{0}(n) \approx \frac{1}{2} \Delta G_{s, \mathrm{dim}}^{0}\left(n-\frac{1}{2}\right)^{2}
$$

Thus, in this ideal system the increment in strain free energy is proportionally greater for each additional subunit, resulting in a proportionally greater free energetic barrier for growing longer polymers with a relatively small effect on the formation of shorter polymers. Subsequently, polymer length distributions will become tighter as mean length increases, approaching polymers with a uniform length (Figure 2P and S1). Conversely in systems where addition of subunits contributes less strain with polymer length, which may be achieved with strain-release mechanisms, an increase in variance with length is expected. In addition, length distributions with more complex strain models can be predicted. For example, a molecular version of the Penrose 
subunit, might be well described by a 2-phase model with a low and high strain regime. For shorter polymers, there may be a relatively low strain free energy increment associated with the entropic cost of restraining the motions of regulatory domains. Whereas for longer polymers, where regulatory domains are forced against a physical border, further extension will be associated with a greater strain free energy increment.
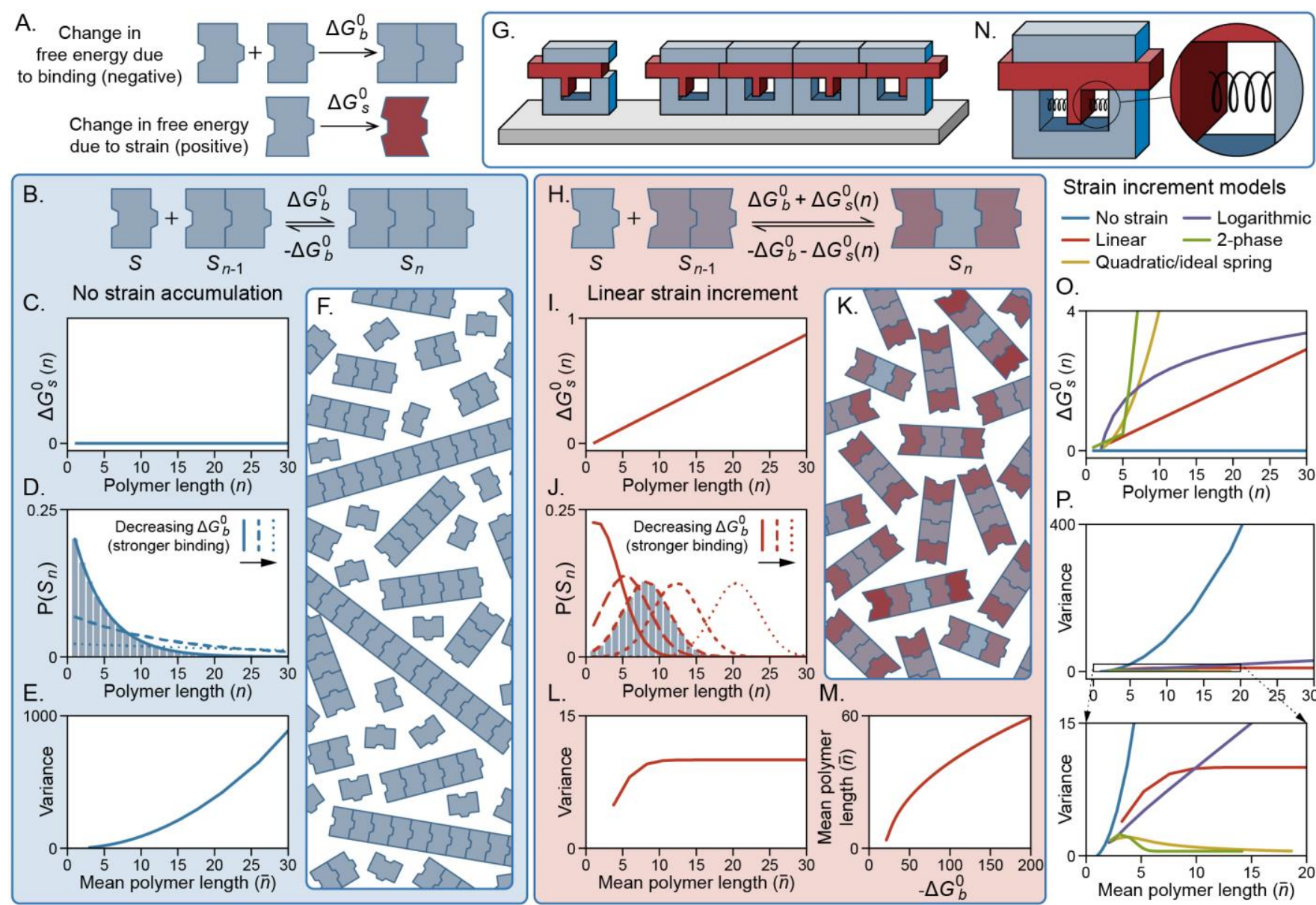

Strain increment models - No strain — Logarithmic - Linear - 2-phase — Quadratic/ideal spring 0 .
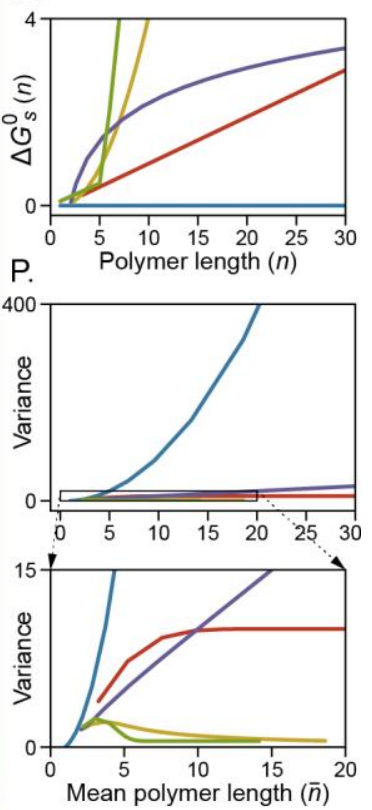

Figure 2 - Thermodynamic modeling of different polymerization schemes. (A) Descriptions of $\Delta G_{b}^{0}$ and $\Delta G_{s}^{0}$ as they pertain to subunit binding and strain. (B) Depiction of a system of 'passively' polymerizing subunits without strain accumulation as shown in graph in (C). (D) Predicted polymer length distribution from thermodynamic model of the non-strain accumulating system with a total subunit concentration of $20 \mathrm{nM}$ and different values of $\Delta G_{b}^{0}(-20.7,-23.0$ 
and $-25.6 R T \cdot \mathrm{mol}$ ). (E) Plot of predicted variance $v s$ mean polymer length from thermodynamic model, which increases exponentially in a non-strain accumulating system. (F) Pictorial representation showing predicted wide length distributions from passive polymerization. (G) A "blocking device" described by Penrose ${ }^{76}$ which prohibits the addition of subunits once a maximum polymer length has been reached. In this depiction, binding domains are blue and the sliding "blocking" domain is red. (Diagram adapted from Ref. $\left.{ }^{76}\right)(\mathrm{H})$ Depiction of a system of subunits that polymerize with strain accumulation. (I) A graph showing linear strain increment with polymer length. (J) Predicted polymer length distributions of a system with linear strain increment, a total subunit concentration of $20 \mathrm{nM}, \Delta G_{s, \mathrm{dim}}^{0}=0.1 \mathrm{RT} \cdot \mathrm{mol}$ and different values of $\Delta G_{b}^{0}(-20.7,-23.0,-25.3,-29.9$ and $-43.7 R T \cdot \mathrm{mol})$ from thermodynamic modeling. $(\mathrm{K})$ Pictorial representation showing relatively narrow polymer length distributions for a strain accumulation scheme. (L) Graph showing predicted variance vs mean length for subunits that polymerize with linear strain increment. Variance is far smaller than that of non-strain accumulation systems and is constant for sufficiently large polymers. (M) Increasing binding strength by decreasing values of $\Delta G_{b}^{0}$ at a fixed concentration cause mean polymer lengths to increase sublinearly. (N) The addition of ideal springs to the subunits in $G$ result in a quadratic strain increment with subunit addition. (O) A comparison of various strain increment models, including from an ideal spring with $\Delta G_{s, \mathrm{dim}}^{0}$ as the spring constant resulting in a quadratic strain increment $\left(\Delta G_{s}^{0}(n)=\frac{1}{2} \Delta G_{s, \text { dim }}^{0}(n-1)^{2}\right)$, a sublinear logarithmic strain increment $\left(\Delta G_{s}^{0}(n)=\right.$ $\left.\ln (n-1) \Delta G_{s, \mathrm{dim}}^{0}\right)$ and a 2-phase model which comprises an initial weak linear strain increment and a stronger strain increment that engages once a certain polymer length has been reached $\left(\Delta G_{S}^{0}(n)=\frac{k_{1}}{\left(1+\frac{k_{1}}{k_{2}}\right) n}\right.$ when $n \leq b+\frac{k_{1}}{k_{2}}$ or $\Delta G_{S}^{0}(n)=k_{2}(n-b)$ when $n>b+\frac{k_{1}}{k_{2}}$, where $k_{1}$ and 
$k_{2}$ are the weaker and stiffer increments respectively and $b$ is the polymer length at which the second spring is invoked). (P) Predicted variances for the strain models described in O, illustrating the vast difference in polymer length variance between strain-accumulating and non-strainaccumulating polymerization schemes. Inset (lower) shows a detailed view of the variance with different strain models. Predicted mean lengths and variance were calculated from theoretical length distributions, which were determined by solving the simultaneous equations (4) with equation (5) for different strain models using a custom solver implemented in Matlab Version $2019 \mathrm{~b}$ (9.7.0.1190202). Parameters used were $\left[S_{\text {total }}\right]=20 n M$ and $\Delta G_{s, \mathrm{dim}}^{0}=k_{1}=0.1 R T$. mol. $k_{2}=2 R T \cdot \mathrm{mol}, b=5$ subunits and a range of values between $-20.7 \mathrm{RT} \cdot \mathrm{mol}$ and $-202.6 R T \cdot \mathrm{mol}$ for $\Delta G_{b}^{0}$. For all strain models, $\Delta G_{s, \mathrm{dim}}^{0}=0.1 R T \cdot \mathrm{mol}$.

\section{Embodiment of strain accumulation polymer into a molecular subunit}

We sought to construct a molecular subunit capable of forming self-limiting polymers with strain accumulation, which like the Penrose model (Figure 2G), utilises the cumulative displacement of a mobile domain. This subunit, which we call the PolyBrick, comprises three connected domains, each a rigid bundle of 28 or 30 double-stranded DNA (dsDNA) duplexes (Figure 3A-C and S2). These bundles are arranged to create a pair of allosteric 'blocking' domains that are flexibly linked to a central 'binding' domain via short duplexes that protrude perpendicularly from the helical axis of the rigid domains. These linkers act as hinges and allow each domain to move relative to each other in an arc approximating a sliding translation in the direction of polymerisation (Figure 3D). The central binding domain has a fixed length and the ends of the duplexes present short oligonucleotide extensions (plugs) and truncations (sockets) so that one side forms a complementary binding site to the other side, thus allowing polymerisation (Figure 3E). The 
sequence of each binding site therefore depends on the sequence of the scaffold strand at the socket. Consequently, all combinations of plug/socket sites used in this study were unique and designed to prevent binding in unintended orientations. Inter-subunit binding free energies $\left(\Delta G_{b}^{0}\right)$ can be controlled by altering the number and length of plugs and sockets. Plug/sockets can also be removed completely or replaced with poly-thymine (poly-t) oligonucleotide extensions to prevent polymerisation. The two blocking domains have poly-t extensions from both ends of the duplexes to prevent binding via the blocking domains and to ensure that they are longer than the central binding domain (Figure 3F). This difference in length consequently requires that binding via the central domain causes displacement of the blocking domains (Figure 3G). In principle, the magnitude of the displacement increases linearly with the number of subunits in the polymer up to a maximal distance dictated by the linker length. The displacement distance per monomer can be readily tuned by altering the length of poly-t's thereby increasing the fundamental strain energy $\left(\Delta G_{s, \mathrm{dim}}^{0}\right)$ (Figure 3G,H). It is possible that with sufficient strain free energy, other displacement mechanisms may be invoked such as the breaking of base pair interactions. This may release strain and result in longer polymers than are allowable by the linker length.

We also constructed a control subunit ("control brick"), designed to polymerize without strain accumulation (Figure 3I and S3). Flexible linkers were removed to eliminate allostery, resulting in a rigid structure. Consequently, all binding sites on control bricks were equivalent regardless of the polymer length. The central binding domain of the control brick was structurally the same as the PolyBrick, but the blocking domains were designed to be shorter to minimize any impact on binding affinities. It was not sufficient to simply remove poly-t extensions from the blocking domains because the resulting non-passivated blunt ends can bind to neighbouring subunits via base-stacking interactions. ${ }^{4,17,40}$ 


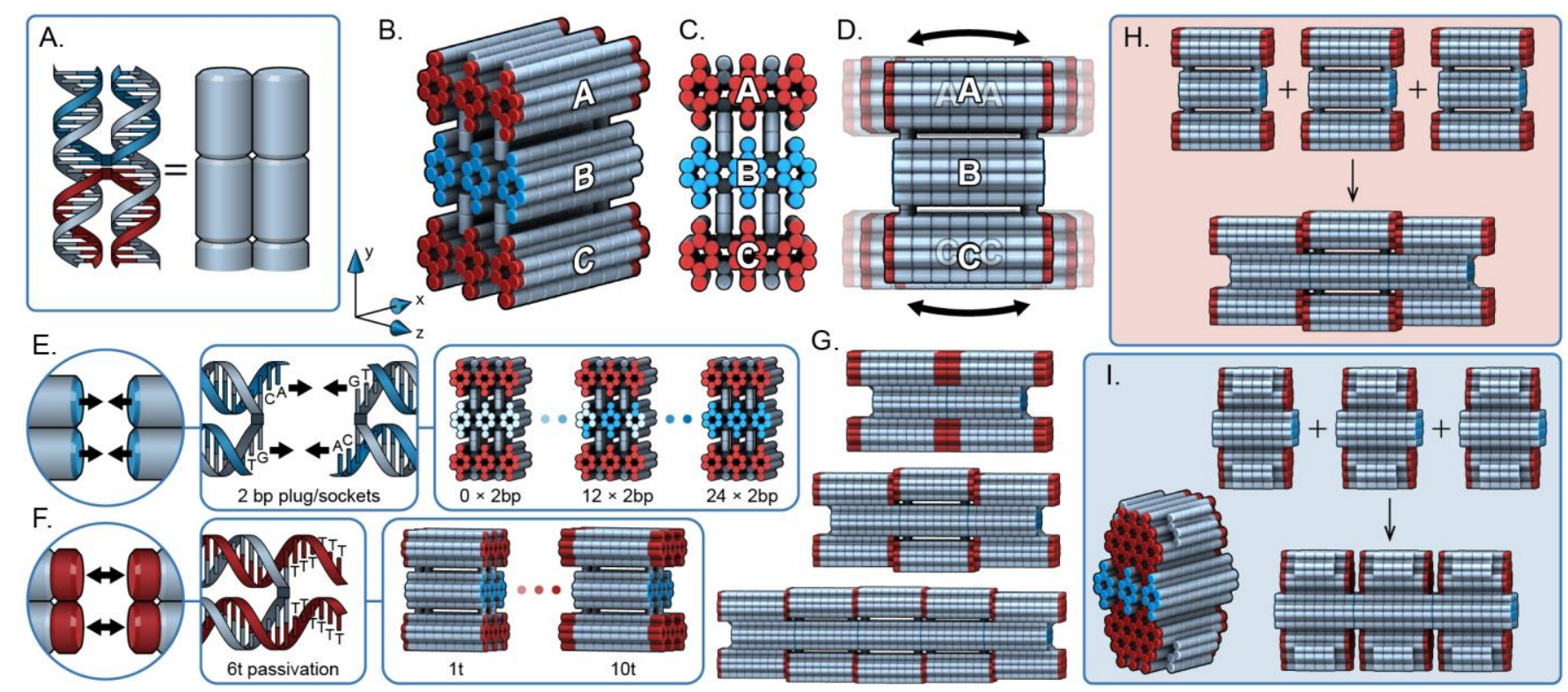

Figure 3 - Design of DNA origami PolyBrick and control brick. (A) dsDNA duplexes are represented as solid cylinders. (B) The design of the PolyBrick subunit comprises three connected domains labelled A, B and C. The B domain has a binding interface (blue) on the front and rear, so that the front of the subunit is complementary to the rear. The A and $\mathrm{C}$ domains have passivated interfaces (red), which are designed not to bind. (C) The three domains are connected with flexible linkers, shown vertically. (D) The linkers allow movement of the A and C domains relative to the B domain in an arc approximating a linear sliding motion. (E) The ends of DNA duplexes on binding domains comprise oligonucleotide extensions (plugs) and truncations (sockets) that bind to form 2 base-pair (bp) interactions. By adjusting the number of plug/socket interfaces, the strength of the binding site is adjustable from $0 \times 2$ bp (no plugs, only sockets) to $24 \times 2$ bp (all possible plug/socket interactions). All plug/socket sequences are shown in Supplementary Figure S2. (F) The ends of DNA duplexes on blocking domains are passivated with poly-thymine oligonucleotide extensions. The size of these domains, and hence the rate of strain accumulation 
can be adjusted by changing the length of the staple extensions from 1t (smallest) to 10t (largest).

(G) Schematic depiction illustrating how longer poly-t extensions result in a larger displacement of blocking domains and hence larger strain free energy per polymer length. Altering the length of poly-t extensions thus provides a means to control mean polymer length: PolyBricks with longer poly-t extensions (top) should produce shorter polymer lengths than PolyBricks with shorter polyt extensions (bottom). (H) Schematic of three PolyBrick monomers binding to form a strained polymer. (I) Design of the non-strain-accumulating control brick (left) and schematic showing formation of an unstrained 3-subunit polymer (right).

\section{Structural properties of PolyBrick Monomer}

We assessed the in-solution properties of the PolyBrick with poly-t extension lengths of $1 \mathrm{t}, 3 \mathrm{t}$ and 6t, by performing molecular dynamics (MD) simulations with the oxDNA coarse-grained model. ${ }^{77}$ The oxDNA model has been used to characterise a wide variety of DNA nanostructures ${ }^{78-}$ 81 and is well suited for elucidating the structural and dynamic properties of the PolyBrick. The model provides a good description of the microscopic behaviour base-pairing interactions ${ }^{82}$ and the mechanical properties of single and double stranded DNA, ${ }^{83}$ as well as accurate in solution measurements on the structure and thermal fluctuations of large DNA origami structures ${ }^{81,84}$ and DNA origami with mechanically compliant hinges. ${ }^{85-86}$ In addition, the oxDNA model has been used to characterise the physical processes associated with the force-induced unravelling of DNA origami structures. ${ }^{87}$

Snapshots from MD simulations and plots of translational and angular displacement distributions along x-, y- and z-axes (defined in Supplementary Note 3 and Figures S4-5) for the 
PolyBricks with $1 \mathrm{t}$ extensions are shown in Figure 4A and B respectively. These displacement distributions capture the mean position and thermal fluctuations of blocking domains relative to the central binding domain. The $\mathrm{x}$ - and $\mathrm{y}$-axes were defined by the direction of the helices and the axis that crosses all three domains respectively. Data for all PolyBrick configurations are shown in Figure S6. Displacement distributions were well described by a Gaussian function, which suggests that the free energy for displacement of blocking domains increases quadratically with distance and symmetrically from the mean. The PolyBrick is on average asymmetrical where one end of each of the blocking domains appeared to be further from the central binding domain than the other. Angular displacement distributions around the z-axis $(\Delta \theta z)$ illustrate how the angle of the top and bottom flanking domain were mirrored around that of the central domain. On average, these were displaced by about \pm 10 degrees from the central binding domain and rarely sampled a conformation where domains are aligned. Hence, the PolyBrick appeared mostly as an arrowshaped subunit and this occurred irrespectively of the length of poly-t extensions (Figure 4A).

Visual inspection of the PolyBrick structure revealed that the angular displacement from the central binding domain results from base-stacking interactions between linking duplexes and the ends of duplexes that anchor the linkers to the rigid domains. To accommodate this, the ends of the anchoring helices bend away from the rigid domains at the nearest staple crossover. Subsequently, linkers were extended by the number of base pairs between the end of the anchoring helix and the nearest crossover (Figure S7). The mean angular and translational displacement in all other axes were also offset from the central binding domain (Figure 4B). These non-zero mean displacements likely resulted from a complex combination of factors including those resulting from the base stacking interaction described above. 
To verify predicted structural properties of the PolyBrick oxDNA simulations, we imaged fully passivated monomeric subunits with transmission electron microscopy (TEM). PolyBricks were generally visible as monomeric structures consisting of three roughly parallel rectangular domains with visible striations consistent with the DNA origami honeycomb lattice used ${ }^{6}$ and dimensions that matched our design when viewed along the z-axis (Figure 4C and S8). Also visible were PolyBricks oriented with the $x$ - and y-axes normal to the imaging plane (Figure 4D), however these orientations were far less frequent, presumably because the z-axis orientation maximised contact with the carbon surface. This orientation allowed all three domains to be clearly visualized for most subunits. Consistent with oxDNA simulations, we observed substantial heterogeneity in both $\Delta x$ and $\Delta \theta z$ displacements with many particles adopting the predicted arrow-shaped configuration (Figure S8). Moreover, automated 2D classification of particle shapes yielded an average image resembling that observed in the equivalent orientation in oxDNA simulations (Figure 4E and S9).

Fluctuations were significant across all displacement modes (Figure 4B and S6), suggesting multiple possible deformation mechanisms to accommodate strain were possible, which may not be captured by displacement in one dimension only. Thus, we performed umbrella sampling MD simulations where blocking domains were displaced by different distances along the $\mathrm{x}$-axis (Figure 4B), which corresponds to the expected displacement associated with the binding of subunits (Figure 3H). Fluctuations were used to generate a plot of the unbiased strain free energy of a monomer as a function of the displacement distance of blocking domains $\left(\Delta G_{s, \text { mon }}^{0}(\Delta x)\right)$, using the weighted histogram analysis method (WHAM) ${ }^{88}$ (Figure S10). We observed a relatively lowstress and a high-stress regime. In the low-stress regime, the strain free energy increased quadratically with displacement distances up to a maximum distance allowed by a fully extended 
linker $\left(\Delta G_{s, \text { mon }}^{0}(\Delta x) \propto \Delta x^{2}\right.$ for $\left.-6 \mathrm{~nm}<\Delta x<6 \mathrm{~nm}\right)$ (Figure 4F). Larger displacement distances are associated with a greater increase in strain free energy cost (high-stress regime) and require structural changes other than the free motions of the linker.

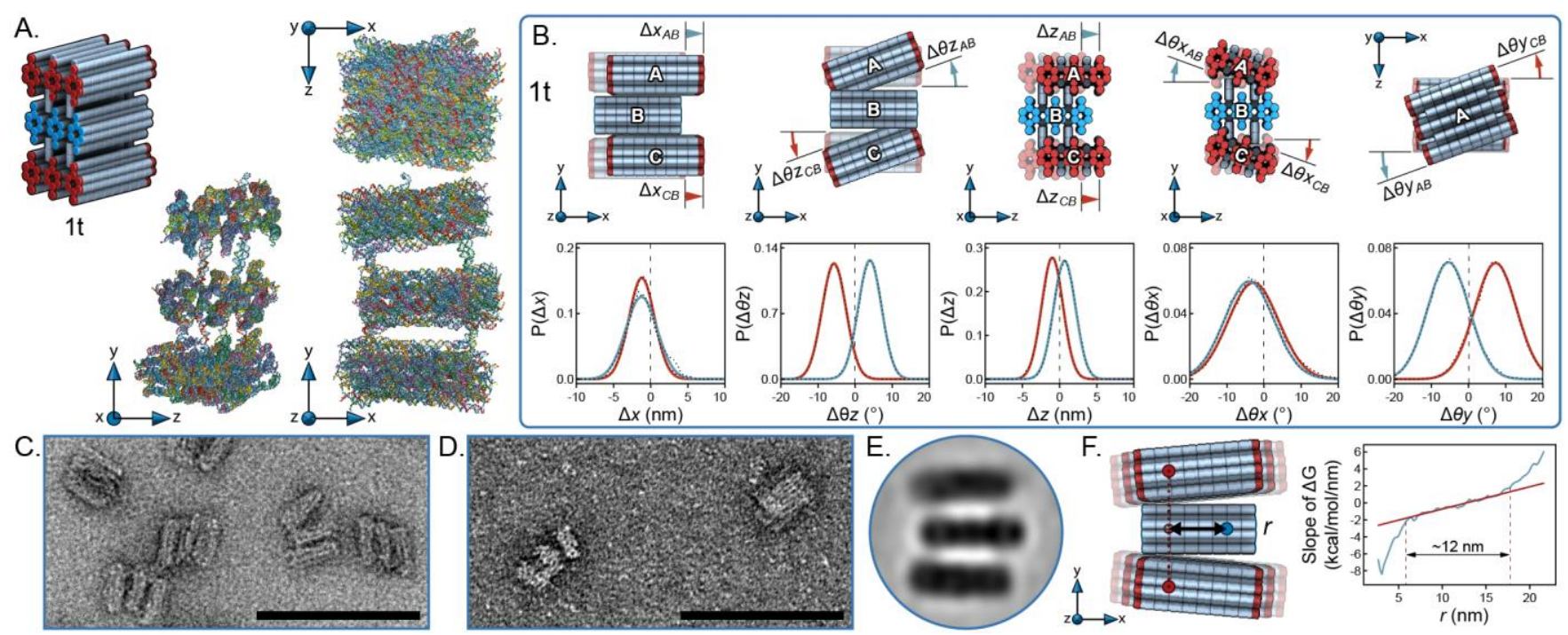

Figure 4 - oxDNA simulations of PolyBrick monomers and TEM micrographs. (A) Left: 3D rendering of a monomeric 1t PolyBrick subunit. Right: representative single frame renderings from oxDNA simulation of the subunit at equilibrium shown in different orientations. (B) Definitions and histograms of fluctuations of the A blocking domain relative to the binding domain in simulations. Data (dotted lines) have been fit to Gaussian distribution (solid lines). Complete data showing fluctuations for domains with different poly-t lengths is shown in supplementary figure S6. (C) Exemplary transmission electron micrograph showing fully passivated PolyBrick monomers oriented with the z-axis normal to the viewing plane. (D) Electron micrograph showing PolyBricks oriented with the $\mathrm{x}$ - and $\mathrm{y}$-axis (left and right, respectively) normal to the viewing 
plane. (E) 2D average of PolyBrick particles oriented as in C. (F) Left: Description of $r$ measurement used in umbrella sampling. Right: Differential free energy profile from oxDNA umbrella sampling (blue) fitted with a straight line (red). There is a $\Delta x$ range of approximately 12 $\mathrm{nm}$ for which the free energy is well approximated by a quadratic fit. Scale bars in C and D are $100 \mathrm{~nm}$.

oxDNA simulations of polymers

To assess the effect of polymerisation on the displacement of the blocking domains, we performed equilibrium MD simulations with oxDNA on PolyBrick polymers that were constrained to lengths of 2- to 9-subunits, and with 1, 3 or 6 thymine extensions on blocking domains (Figure 5A and S11-S27). $\Delta x$ displacement distributions of blocking domains for each subunit within different length polymers (Figure 5B) show how the mean displacement distance increased along the length of the polymer, to a maximal distance of $\sim \pm 7 \mathrm{~nm}$ from the mean position of a monomer subunit (Figure 5C and S28). For 1t polymers, and $3 \mathrm{t}$ and $6 \mathrm{t}$ polymers shorter than 8 and 6 subunits respectively, $\Delta x$ was the dominant mode of displacement and the maximal $\Delta x$ displacement could be accommodated without significant displacements in other modes $(\Delta \theta y, \Delta \theta x, \Delta \theta z$ and $\Delta z)$ (Figures S12-S27). These polymers were in a 'low-stress' regime where strain from polymerisation primarily acted to restrain the free motions of blocking domains. In the low-stress regime, the mean $\Delta x$ displacement distances increased approximately linearly and symmetrically from the centre of the polymer (Figure 5C and S28) as expected from an ideal system depicted in Figures $1 \mathrm{H}$ and $3 \mathrm{H}$. There was a small but observable deviation from linearity in longer polymers where the displacement per subunit was proportionally higher towards polymer ends. The $\Delta x$ displacement distance per subunit decreased sub linearly with polymer length and was larger for 
polymers with longer poly-t extensions as expected and with a narrowing gap with increasing polymer length (Figure 5D and S28). By summing the strain free energy associated with $\Delta x$ displacement distances of each subunit in a polymer, we see that the total strain free energy increases approximately quadratically and more rapidly with increasing poly-t length (Figure 5E and Figure S29). As discussed above, this quadratic increase in total strain free energy is consistent with the thermodynamic model for a linear increment in strain free energy with subunit addition (Equation 8). We note however that the absolute values presented in Figure 5E likely underestimate the true strain free energy because these do not account for interdomain repulsions, the free energy associated with strain release in other modes or an observed decrease in the fluctuation standard deviations as blocking domains were increasingly restrained with increased polymer length. These standard deviations approached a minimum at around $1 \mathrm{~nm}$ for the $1 \mathrm{t}$ PolyBrick. With increasing poly-t length we observe a more rapid decrease in standard deviation with polymer length but the minimum standard deviation decreased only slightly (Figure S30). Blocking domains behaved differently in polymers that were forced to be longer than can be accommodated for by the linker length ( $>8$ for $3 \mathrm{t}$ and $>6$ for $6 \mathrm{t}$ PolyBricks). We refer to these longer polymers as being in a 'high-stress' regime, in which we observe additional displacement modes where the blocking domains of some subunits are laterally displaced from the polymer axis resulting in a large $\Delta \theta z$ (Figures S12-S27). This lateral displacement allows blocking domains of neighbouring subunits to reset their $\Delta x$ displacement effectively providing a displacement release mechanism (Figure 5A-bottom) and a subsequent discontinuity in the mean $\Delta x$ displacement distances (Figure 5C and S28) and the $\Delta x$ strain free energy (Figure 5E). Importantly however, this break in $\Delta x$ displacement does not necessarily equate to a break in the accumulation of total strain free energy, which accounts for deformations in all possible modes. 
The $\Delta x$ displacement in response to polymerisation observed in oxDNA simulations differ from expectations from a macroscopic version of the Penrose subunit in Figures $2 \mathrm{G}$ and $2 \mathrm{~N}$. Macroscopic subunits with hard boundaries between subunits represent a hard, mechanical limit where we expect the slope of the $\Delta x$ displacement to be constant and equal to the difference in length between blocking and binding domains. On a molecular scale however, the blocking domains of the PolyBrick are subject to thermal fluctuations and 'soft' repulsions from poly-t extensions (Figure 5F,G). Consequently, the displacement of blocking domains in response to polymerisation on a molecular scale likely results from a complex interplay of multiple physical factors.

To better define our expectations for the $\Delta x$ displacement of blocking domains and strainaccumulation with polymer length in an ideal molecular system, we explored the consequence of thermal fluctuations and soft repulsions in a minimal one-dimensional (1D) model with Monte Carlo (MC) simulations. With thermal fluctuations but hard boundaries between subunits, mean $\Delta x$ displacements are linear (Figure S31). As with oxDNA simulations, the slope corresponds with the displacement per subunit and decreases non-linearly with polymer length, which approaches the hard, mechanical limit defined by $T=0 \mathrm{~K}$, but also with a corresponding decrease in standard deviation. Unlike with oxDNA simulations however, the minimum standard deviation appeared to decrease substantially with increasing poly-t lengths (Figure S31). To capture the effects of soft repulsions between poly-t extensions in the 1D model (Figure 5G, bottom), we performed pulling simulations with oxDNA and integrated the force-distance curve to yield free energy as a function of distance between blocking domains with different length poly-t extensions (Figure 5H). These free energy profiles were fit with a de Gennes form for a polymer brush ${ }^{89-90}$ with the brush thickness as the fitting parameter, which increased with poly-t length as expected. The subsequent 
effect on the displacement of blocking domains was consistent with observations from oxDNA simulations. First, the slope of mean $\Delta x$ displacements decrease with polymer length but with soft repulsions, there was also a slight but observable deviation from linearity in $\Delta x$ displacements for longer polymers (Figure 5I,J). Second, the minimum standard deviation did not decrease substantially with increasing poly-t length (Figure S32). Both observations can be explained physically by the soft boundaries allowing blocking domains to effectively compress their excluded volumes, which is not possible with hard boundaries. The slopes of mean $\Delta x$ displacement distance $v s$ subunit position were marginally higher in MC simulations compared with MD, which was not unexpected because oxDNA simulations allow for strain relief in other dimensions that cannot be accounted for in a 1D model. Consequently, the total strain free energy from mean $\Delta x$ displacement distances were also greater in MC simulations compared with oxDNA simulations (Figure 5K). Nonetheless, MC simulations demonstrate how the $\Delta x$ displacement of blocking domains in response to strain accumulation from polymerisation, is well explained by accounting for not only the excluded volume, but also thermal fluctuations and soft repulsions between blocking domains. 


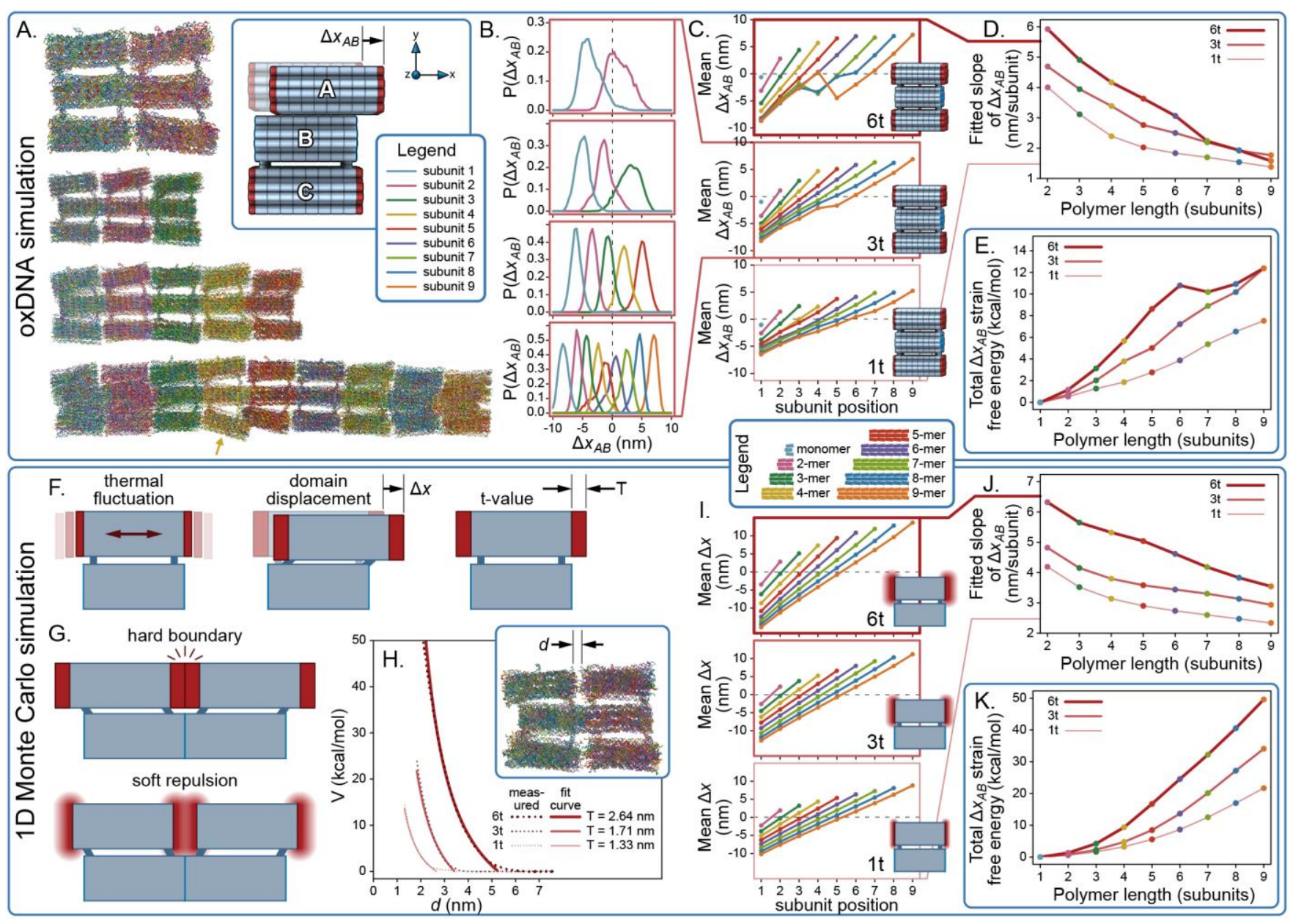

Figure 5 - Simulations of PolyBrick polymers. (A) Representative single frame renderings from oxDNA simulations of $3 \mathrm{t}$ PolyBrick polymers ranging from 2 -subunits (top) to 9 -subunits long (bottom) in solution. Arrow indicates a blocking domain that has been displaced laterally. (B) Frequency histograms of the position of blocking domain A relative to binding domain B along the $\mathrm{x}$-axis $\left(\Delta x_{A B}\right)$ for each subunit in polymers ranging from 2- (top) to 9-subunits (bottom). (C) Means of $\Delta x_{A B}$ plotted against subunit position in polymers ranging from 2- to 9-subunits long for PolyBricks with 1t, $3 t$ and $6 t$ designs. (D) Fitted slopes of the data shown in C. (E) Calculated total strain free energies for the displacement associated with $\Delta x_{A B}$ in oxDNA simulations of different polymer lengths and PolyBrick variants. (F) Description of 1-dimensional Monte Carlo simulation parameters including thermal fluctuation (left), Domain displacement (middle) and poly-t length 
(right). (G) The algorithm was configured to simulate either hard boundaries (top) or soft repulsions (bottom) between blocking domains. (H) Parameters for the soft repulsion parameters for different poly-t values were calculated from oxDNA simulations, fitting measurements to a de Gennes form for a polymer brush. (I) Mean $\Delta x$ displacements plotted against subunit position in polymers ranging from 2- to 9-subunits long for PolyBricks with 1t, 3t and 6t designs. (J) Fitted slopes of the data shown in I. (K) Calculated total strain free energies for the displacement associated with $\Delta x$ in Monte Carlo simulations of different polymer lengths and PolyBrick variants.

\section{Experimental length distributions for PolyBrick subunits}

We synthesized PolyBricks in various configurations, differing by the number of plug/socket sites on binding domains $(0,10,14,18$ and $24 \times 2 \mathrm{bp})$ as well as the length of poly-t extensions on either side of each blocking domain (1t, 3t, 6t and 10t). These assemblies were imaged with TEM and the length of polymers measured. Linear polymers were observed where all three domains of each subunit were clearly visible (Figure 6A) providing a clear measure of polymer length. Some polymeric particles however, appeared malformed, where either some domains were not visible or where it was difficult to determine whether binding occurred specifically via the central domain. It was impossible to discern whether this was a consequence of surface immobilisation and staining with uranyl acetate, or from the orientation of the polymer. Thus, polymer lengths were obtained only from particles for which we could directly observe the mechanism of binding to obtain experimental length distributions $(\sim 73 \%)$ (Figure $6 \mathrm{~B})$. All other particles were ignored (Supplementary Note 4 and Figure S33). For comparison, we also measured length distributions on the control brick, which was void of mechanisms for strain accumulation (Figure 6C,D). For control bricks, blocking domains were passivated with 6 t extensions and length distributions were 
also measured for configurations with different numbers of plug/socket sites $(0,4,8,12,16,20$ and $24 \times 2$ bp, Figure $6 \mathrm{C}$ and S3).

Experimental polymer length distributions were numerically fitted with the thermodynamic model for polymerisation with a linear strain increment (Equation 8) in keeping with estimates of strain free energy accumulation from MC and MD simulations. We performed a semiglobal 2parameter fit where $\Delta G_{s, \mathrm{dim}}^{0}$ was fixed for each poly-t length and $\Delta G_{b}^{0}$ was independently fit for each dataset. Fitted distributions agreed well with experimental data (Figure 6B and S34-S60). Inferred $\Delta G_{s, \text { dim }}^{0}$ increased with poly-t length as expected $(0.225,0.233,0.363$ and $0.461 \mathrm{~kJ} / \mathrm{Mol}$ for $1 \mathrm{t}, 3 \mathrm{t}, 6 \mathrm{t}$ and $10 \mathrm{t}$ respectively) (Figure 6E). The relatively small increase in $\Delta G_{s, \mathrm{dim}}^{0}$ for $1 \mathrm{t}$ and $3 t$ extensions suggests that poly-t extensions had a more dramatic effect with longer poly-t lengths, which is consistent with the fitted de Gennes polymer brush thickness (Figure 5H). Mean lengths of PolyBrick assemblies were shorter than those of control bricks as expected and did not reach the number of subunits required to fully extend the linker. This suggests that translation along the $\mathrm{x}$-axis was the dominant mode of displacement and is consistent with data being well described by a linear strain accumulation model.

For control bricks, the fitted $\Delta G_{s, \text { dim }}^{0}$ using the same strain model was substantially lower than for all configurations of the PolyBrick $(0.013 \mathrm{~kJ} / \mathrm{Mol})$ (Figure $6 \mathrm{E})$. The fact that $\Delta G_{s, \mathrm{dim}}^{0}$ was nonzero for control bricks may be due to several factors. First, the number of monomeric control bricks was consistently less than expected. This may be because imaging conditions for control bricks yielded lower resolution micrographs. Subsequently it was difficult to observe structural features in monomeric subunits, which in turn made it difficult to identify monomer subunits over background (Figures S54-S60). Second, since a larger number of particles are required to obtain 
accurate length distribution of longer polymers, histograms of length distributions in control bricks with higher affinities may be undersampled. Indeed, control brick length distributions can also be satisfactorily modelled with geometric length distributions, which appear to better account for the observed longer polymers (Figure S61). An alternative explanation is the presence of defective subunits, which for example, may be missing some plug/socket sites and prematurely terminate polymer growth. Nonetheless, when fit with the same strain model, $\Delta G_{s, \mathrm{dim}}^{0}$ was $\sim 17$ to 35 -fold lower in control bricks than in PolyBricks.

Figure $6 \mathrm{~F}$ shows variance $v s$ mean length calculated from fits of the strain accumulation model to length distributions of control bricks and PolyBricks. As predicted by the thermodynamic model above (Figure 2M), the variance for PolyBricks show sublinear growth with mean polymer length and tend to a constant determined by the poly-t length. Thus, PolyBrick length distributions are consistent with a continuous linear increment in strain accumulation (Figure 2H-L) with no evidence of strain release or alternative strain accumulation mechanisms (Figure 2P). The variance for control brick assembly lengths are larger than for all PolyBrick assemblies of the same mean length and increase approximately linearly with length for the control brick. Thus, strain accumulation in the PolyBricks provides a dramatically greater control over polymer length distributions than the passively polymerising control bricks. Moreover, due to the undersampling of longer polymers, we interpret the observed variance to be a lower limit of the true variance of control bricks. A comparison with estimates of variance $v s$ mean length for the same data fitted to a geometric distribution show a more dramatic difference in variance compared with PolyBricks (Figure S62) where as predicted, variance for a geometric distribution increases quadratically with mean length. 
For both control bricks and PolyBricks, binding free energies decreased linearly with increasing number of plug/sockets in the binding domain (Figure S63). This relationship is expected for wellaligned binding sites on a rigid central binding domain. The slopes for PolyBricks were similar and appeared independent of poly-t length. We estimate from this that each $2 \mathrm{bp}$ plug/socket site contributes a binding free energy of approximately $\Delta \Delta G_{b}^{0}=-0.21 \pm 0.02 \mathrm{~kJ} / \mathrm{mol}$. The $\mathrm{y}-$ intercepts were also consistent: $\Delta G_{b}^{0}=-46.71 \pm 0.48 \mathrm{~kJ} / \mathrm{mol}$. Interestingly, the relatively low contribution to the binding energy of each plug/socket site indicates that each plug socket site alone contributes very little binding free energy. Moreover that the intrinsic affinity between subunits is substantial, which with no plug/socket sites corresponds to a dissociation constant of $K_{d} \approx 3 \mathrm{nM}$. The slope for control bricks was less steep with each $2 \mathrm{bp}$ plug/socket domain contributing $-0.13 \mathrm{~kJ} / \mathrm{mol}$. The reason for this difference in affinity between PolyBrick and control subunit is unclear, but could be due to difference in nucleotide sequences at the plug/sockets or differences in structural rigidity of the binding domains. ${ }^{41}$

To gain a more detailed understanding of the mechanisms for self-limiting polymer growth, we performed steered MD simulations to measure the force required to pull bound subunits apart (Figure S64). This was unaffected by poly-t length suggesting that the fundamental strain energy $\Delta G_{s, \mathrm{dim}}^{0}$, has little effect on the dissociation rate of subunits and rather that increasing strain energy reduces the association rates. Indeed, in contrast to dissociation, we observe that the force required to pull subunits together increases with poly-t length (Figure S65). These data are consistent with a mechanism whereby subunit binding requires flanking domains to be spontaneously displaced sufficiently to allow contact between binding domains. As polymers grow, sufficient displacement becomes decreasingly favourable as the entropic cost for binding increases. The mechanism has some interesting implications. First, there may be a limit in the extent to which mean polymer 
length can be increased by increasing affinity between subunits. This is because the spontaneous displacement of flanking domains will eventually present an insurmountable kinetic barrier to polymer growth regardless of whether growth is thermodynamically favoured. Second, increasing affinity may instead lead to progressively tighter length distributions. As dissociation rates approach zero polymer lengths will be entirely kinetically limited by the degree to which flanking domains are spontaneously displaced. Moreover, as dissociation rates decrease, polymers will be increasingly stable. 

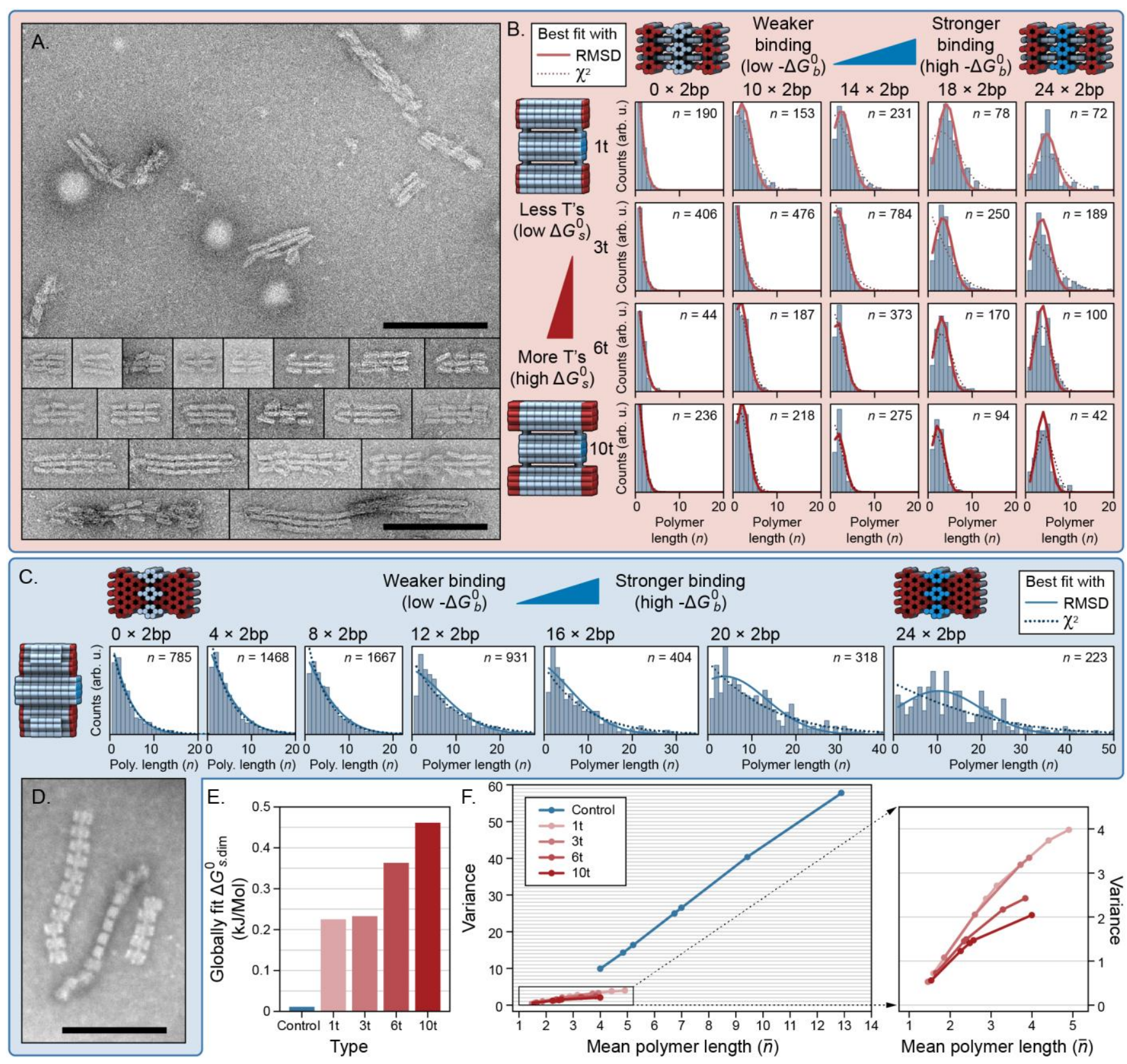

Figure 6 - Measurements of PolyBrick polymers from TEM images. (A) Top: Exemplary TEM micrograph of PolyBrick with $18 \times 2 \mathrm{nt}$ binding domain and $6 \mathrm{t}$ blocking domains. Bottom: Various exemplary TEM micrographs of PolyBrick polymers, ranging from 2- to 15-subunits in length. (B) Histograms of polymer length measurements with RMSE (bold) and $\chi^{2}$ (dotted) fits from thermodynamic model for linear strain accumulation for PolyBrick design variants with binding 
domain configurations ranging from 0 to $24 \times 2 \mathrm{bp}$ (left to right) and blocking domain configurations ranging from 1 t to 10 t (top to bottom). (C) Histograms of polymer length measurements with RMSE (bold) and $\chi^{2}$ (dotted) fits from thermodynamic model for linear strain accumulation for the control brick, with binding domain configurations ranging from 0 to $24 \times 2$ bp (left to right). (D) Exemplary TEM micrograph of control bricks with $8 \times 2$ bp binding domain. (E) $\Delta G_{s, \mathrm{dim}}^{0}$ values for global fits to experimental data for all PolyBricks and control brick variants. (F) Left: Plot of variance to mean polymer length from histogram fits of TEM data for all PolyBrick and control brick variants. Right insert: Enlarged section showing PolyBrick variants. Scale bars in A and D are $200 \mathrm{~nm}$.

\section{CONCLUSIONS}

We offer a theoretical explanation of how strain accumulation can be used to control the mean length and variance of self-assembling polymers as a function of the binding free energy and the fundamental strain free energy. Experimentally, we designed and synthesised the molecular PolyBrick subunit using the DNA origami method to demonstrate the use of strain accumulation via geometric frustration as a rational design principle. We show how PolyBrick subunits selfassemble into polymers with length distributions that are consistent with theoretical expectations. In addition, we demonstrate how length distributions can be rationally tuned by altering the plug/socket or poly-t extension configurations, which control the binding free energy and fundamental strain free energy respectively.

Despite the many advances in our understanding of DNA nanostructures, detailed characterisation of the PolyBrick's structure and dynamics with oxDNA MD simulations revealed many properties that were not obvious from its design. These properties included the intrinsic asymmetry of the subunits, the many possible displacement modes for strain release and the free 
energy associated with displacement of allosteric domains. In addition, we demonstrate using MC simulations that compared with a macroscopic system, fundamentally different physical forces dominate strain accumulation on a molecular scale. These forces include thermal fluctuations and in the case of the PolyBrick subunit, 'soft' boundaries between subunits. Our results define expectations for both, which is particularly important for future nanoscale design. Finally, both MC and MD simulations provided estimations of how strain accumulates for different configurations of the PolyBrick subunit, which were broadly consistent with experimental measurements.

Given the near limitless possibilities in subunit designs afforded by the DNA origami method, there is considerable scope for further exploration and application in DNA nanotechnology. For example, linear aggregation with alternative strain accumulation rates could be explored to obtain tighter length distributions that approach uniformity, as well as alternative designs that do not allow for strain release via the lateral motion of allosteric domains. DNA origami subunits could also utilise geometric frustration to explore existing theoretical models such as for controlling of the aspect ratio and length scales of $2 \mathrm{D}$ structures by the rationally altering strain energies and surface tensions. ${ }^{91}$

\section{METHODS}

\section{Simulations}

All molecular dynamics simulations were performed using the oxDNA coarse-grained model with a time-step of 0.005 in simulation units, which corresponds to a time-step of $15 \mathrm{fs}$ in real units, a 
temperature of $300 \mathrm{~K}$ using the Langevin thermostat, a damping coefficient corresponding to a diffusion coefficient of 2.5 simulation units and a salt concentration of $[\mathrm{Na}+]=1.0$ M. All initialization files are available on the Oxford Research Archive data repository (https://ora.ox.ac.uk/objects/uuid:90bf857c-06e3-4274-bba3-a180f729fbe5). Monte Carlo simulations were performed using custom code. See Supplementary Information for more detailed descriptions of oxDNA molecular dynamics simulations and Monte Carlo simulations.

\section{DNA origami synthesis}

DNA origami structures were designed using Cadnano ${ }^{92}$ and $\mathrm{CanDo}^{93}$ iteratively. Scaffold strands (20 nM unless otherwise specified, M13mp18, Bayou Biolabs) were mixed with a 5-fold excess of staple strands (Integrated DNA Technologies) in buffer with $5 \mathrm{mM}$ Tris, $1 \mathrm{mM}$ EDTA and $22 \mathrm{mM} \mathrm{MgCl} 2$. Structures were heated to $65{ }^{\circ} \mathrm{C}$ for 15 minutes, then cooled from $60{ }^{\circ} \mathrm{C}$ to $40{ }^{\circ} \mathrm{C}$ over 40 hours, then cooled from $40^{\circ} \mathrm{C}$ to $25^{\circ} \mathrm{C}$ over 5 hours. Structures were left to equilibrate, unpurified from excess staples, for 24 hours at room temperature prior to imaging.

\section{TEM imaging}

Samples were drop cast onto carbon/formvar coated copper grids which had been glow discharged for 60 seconds, and given 4 minutes to adsorb. After excess sample was wicked off, a drop of $2 \%$ aqueous uranyl acetate was applied to the grid and wicked off immediately. Imaging of PolyBricks was performed on a Tecnai G2 20 transmission electron microscope (FEI) in bright field mode at $200 \mathrm{kV}$. Imaging of control bricks was performed on a JEOL TEM-1400 transmission electron microscope at $120 \mathrm{kV}$. Micrographs were analysed in Image ${ }^{94}$ by manually measuring structures while blind to assembly condition and design variant. Histograms and fits were created in either Mathematica v11.2 or Matlab. 


\section{SUPPORTING INFORMATION}

Supporting information is available free of charge via the Internet at http://pubs.acs.org. These are incorporated in a single document (PDF) consisting of supplementary notes showing analytical derivations, vector definitions for MD analysis and criteria for particle selection, figures showing additional data and detail on thermodynamic models, DNA origami architecture and Cadnano designs, displacement of blocking domains, electron microscopy micrographs, data from MD and MC simulations and experimentally measured length distributions.

\section{CORRESPONDING AUTHOR}

Lawrence K. Lee, EMBL Australia Node for Single Molecule Science, School of Medical Sciences, UNSW Sydney, 2052, Australia. lawrence.lee@unsw.edu.au

\section{AUTHOR CONTRIBUTIONS}

J.F.B. Conceived the project, designed, synthesized DNA origami structures, performed TEM imaging, processed and analyzed data and wrote the manuscript. C.K.W and J.P.K.D performed MD and MC simulations and analysis, and wrote the manuscript, J.C.B. developed mathematical models, T.E.O. developed thermodynamic models and wrote the manuscript, L.K.L. conceived and coordinated the project, developed thermodynamic models and wrote the manuscript. All authors have given approval to the final version of the manuscript.

\section{FUNDING SOURCES}


This research was supported by the Australian Research Council Centre of Excellence in Synthetic Biology (Grant ID CE200100029) and the National Health and Medical Research Council (Grant ID APP1129234). L.K.L was supported by the Australian Research Council Discovery Early Career Research Award (Grant ID DE140100262) and C.K.W was supported by the Croucher Foundation.

\section{ACKNOWLEDGMENT}

The authors thank the facilities of Microscopy Australia at the Electron Microscope Unit, Mark Wainwright Analytical Centre, UNSW Sydney. We would also like to thank Professor Satoshi Murata for introducing us to the work of L. S. Penrose, and Rokiah Alford for assisting in blinded polymer length measurements. We acknowledge the use of the University of Oxford Advanced Research Computing (ARC) facility and the Cambridge Service for Data Driven Discovery (CSD3). We acknowledge funding sources from the Australian Research Council (Grant IDs CE200100029 and DE140100262), the National Health and Medical Research Council (Grant ID DE140100262) and the Croucher Foundation.

\section{REFERENCES}

1. Seeman, N. C., Nucleic Acid Junctions and Lattices. J. Theor. Biol. 1982, 99, 237-247.

2. Seeman, N. C., DNA in a Material World. Nature 2003, 421, 427.

3. Seeman, N. C.; Sleiman, H. F., DNA Nanotechnology. Nat. Rev. Mater. 2018, 3, 17068.

4. Rothemund, P. W. K., Folding DNA to Create Nanoscale Shapes and Patterns. Nature 2006, 440, 297-302.

5. Douglas, S. M.; Dietz, H.; Liedl, T.; Högberg, B.; Graf, F.; Shih, W. M., Self-Assembly of DNA into Nanoscale Three-Dimensional Shapes. Nature 2009, 459, 414-418. 
6. Dietz, H.; Douglas, S. M.; Shih, W. M., Folding DNA into Twisted and Curved Nanoscale Shapes. Science 2009, 325, 725-730.

7. Nangreave, J.; Han, D.; Liu, Y.; Yan, H., DNA Origami: A History and Current Perspective. Curr. Opin. Chem. Biol. 2010, 14, 608-615.

8. Tørring, T.; Voigt, N. V.; Nangreave, J.; Yan, H.; Gothelf, K. V., DNA Origami: A Quantum Leap for Self-Assembly of Complex Structures. Chem. Soc. Rev. 2011, 40, 5636-5646.

9. Saccà, B.; Niemeyer, C. M., DNA Origami: The Art of Folding DNA. Angew. Chem. Int. Ed. 2012, 51, 58-66.

10. Wang, P.; Meyer, T. A.; Pan, V.; Dutta, P. K.; Ke, Y., The Beauty and Utility of DNA Origami. Chem 2017, 2, 359-382.

11. Hong, F.; Zhang, F.; Liu, Y.; Yan, H., DNA Origami: Scaffolds for Creating Higher Order Structures. Chem. Rev. 2017, 117, 12584-12640.

12. Funke, J. J.; Dietz, H., Placing Molecules with Bohr Radius Resolution Using DNA Origami. Nat. Nanotech 2015, 11, nnano. 2015.240.

13. Ding, B.; Deng, Z.; Yan, H.; Cabrini, S.; Zuckermann, R. N.; Bokor, J., Gold Nanoparticle Self-Similar Chain Structure Organized by DNA Origami. J. Am. Chem. Soc. 2010, $132,3248-3249$.

14. Bui, H.; Onodera, C.; Kidwell, C.; Tan, Y. P.; Graugnard, E.; Kuang, W.; Lee, J.;

Knowlton, W. B.; Yurke, B.; Hughes, W. L., Programmable Periodicity of Quantum Dot Arrays with DNA Origami Nanotubes. Nano Lett. 2010, 10, 3367-3372.

15. Marini, M.; Piantanida, L.; Musetti, R.; Bek, A.; Dong, M.; Besenbacher, F.; Lazzarino, M.; Firrao, G., A Revertible, Autonomous, Self-Assembled DNA-Origami Nanoactuator. Nano Lett. 2011, 11, 5449-5454.

16. Marras, A. E.; Zhou, L.; Su, H.-J.; Castro, C. E., Programmable Motion of DNA Origami Mechanisms. Proc. Natl. Acad. Sci. USA 2015, 112, 713-718.

17. Gerling, T.; Wagenbauer, K. F.; Neuner, A. M.; Dietz, H., Dynamic DNA Devices and Assemblies Formed by Shape-Complementary, Non-Base Pairing 3D Components. Science 2015, 347, 1446-1452.

18. Kuzuya, A.; Ohya, Y., Nanomechanical Molecular Devices Made of DNA Origami. Acc. Chem. Res. 2014, 47, 1742-1749. 
19. Kopperger, E.; List, J.; Madhira, S.; Rothfischer, F.; Lamb, D. C.; Simmel, F. C., A SelfAssembled Nanoscale Robotic Arm Controlled by Electric Fields. Science 2018, 359, 296-301.

20. Singh, J. K. D.; Luu, M. T.; Abbas, A.; Wickham, S. F., Switchable DNA-Origami

Nanostructures That Respond to Their Environment and Their Applications. Biophys. Rev. 2018, $10,1283-1293$.

21. Kuzuya, A.; Watanabe, R.; Yamanaka, Y.; Tamaki, T.; Kaino, M.; Ohya, Y., Nanomechanical DNA Origami Ph Sensors. Sensors 2014, 14, 19329-19335.

22. Nickels, P. C.; Wünsch, B.; Holzmeister, P.; Bae, W.; Kneer, L. M.; Grohmann, D.; Tinnefeld, P.; Liedl, T., Molecular Force Spectroscopy with a DNA Origami-Based Nanoscopic Force Clamp. Science 2016, 354, 305-307.

23. Walter, H.-K.; Bauer, J.; Steinmeyer, J.; Kuzuya, A.; Niemeyer, C. M.; Wagenknecht, H.A., "DNA Origami Traffic Lights" with a Split Aptamer Sensor for a Bicolor Fluorescence Readout. Nano Lett. 2017, 17, 2467-2472.

24. Dutta, P. K.; Zhang, Y.; Blanchard, A. T.; Ge, C.; Rushdi, M.; Weiss, K.; Zhu, C.; Ke, Y.; Salaita, K., Programmable Multivalent DNA-Origami Tension Probes for Reporting Cellular Traction Forces. Nano Lett. 2018, 18, 4803-4811.

25. Schmied, J. r. J.; Forthmann, C.; Pibiri, E.; Lalkens, B.; Nickels, P.; Liedl, T.; Tinnefeld, P., DNA Origami Nanopillars as Standards for Three-Dimensional Superresolution Microscopy. Nano Lett. 2013, 13, 781-785.

26. Raab, M.; Schmied, J. J.; Jusuk, I.; Forthmann, C.; Tinnefeld, P., Fluorescence Microscopy with 6 Nm Resolution on DNA Origami. ChemPhysChem 2014, 15, 2431-2435.

27. Schmied, J. J.; Raab, M.; Forthmann, C.; Pibiri, E.; Wünsch, B.; Dammeyer, T.;

Tinnefeld, P., DNA Origami-Based Standards for Quantitative Fluorescence Microscopy. Nat.

Protoc. 2014, 9, 1367-1391.

28. Korpelainen, V.; Linko, V.; Seppä, J.; Lassila, A.; Kostiainen, M. A., DNA Origami Structures as Calibration Standards for Nanometrology. Meas. Sci. Technol. 2017, 28, 034001.

29. Baker, M. A.; Nieves, D. J.; Hilzenrat, G.; Berengut, J. F.; Gaus, K.; Lee, L. K., Stoichiometric Quantification of Spatially Dense Assemblies with Qpaint. Nanoscale 2019. 30. Rinker, S.; Ke, Y.; Liu, Y.; Chhabra, R.; Yan, H., Self-Assembled DNA Nanostructures for Distance-Dependent Multivalent Ligand-Protein Binding. Nat. Nanotech 2008, 3, 418-422. 
31. Derr, N. D.; Goodman, B. S.; Jungmann, R.; Leschziner, A. E.; Shih, W. M.; ReckPeterson, S. L., Tug-of-War in Motor Protein Ensembles Revealed with a Programmable DNA Origami Scaffold. Science 2012, 338, 662-665.

32. Dunn, K. E.; Dannenberg, F.; Ouldridge, T. E.; Kwiatkowska, M.; Turberfield, A. J.; Bath, J., Guiding the Folding Pathway of DNA Origami. Nature 2015, 525, 82-86.

33. Funke, J. J.; Ketterer, P.; Lieleg, C.; Schunter, S.; Korber, P.; Dietz, H., Uncovering the Forces between Nucleosomes Using DNA Origami. Sci. Adv. 2016, 2.

34. Zhang, H.; Chao, J.; Pan, D.; Liu, H.; Huang, Q.; Fan, C., Folding Super-Sized DNA Origami with Scaffold Strands from Long-Range Pcr. Chem. Commun. 2012, 48, 6405-6407.

35. Marchi, A. N.; Saaem, I.; Vogen, B. N.; Brown, S.; LaBean, T. H., Toward Larger DNA Origami. Nano Lett. 2014, 14, 5740-5747.

36. Wagenbauer, K. F.; Sigl, C.; Dietz, H., Gigadalton-Scale Shape-Programmable DNA Assemblies. Nature 2017, 552, 78.

37. Tikhomirov, G.; Petersen, P.; Qian, L., Fractal Assembly of Micrometre-Scale DNA Origami Arrays with Arbitrary Patterns. Nature 2017, 552, 67.

38. Pfeifer, W.; Saccà, B., From Nano to Macro through Hierarchical Self-Assembly: The DNA Paradigm. ChemBioChem 2016, 17, 1063-1080.

39. Iinuma, R.; Ke, Y.; Jungmann, R.; Schlichthaerle, T.; Woehrstein, J. B.; Yin, P., Polyhedra Self-Assembled from DNA Tripods and Characterized with 3D DNA-Paint. Science 2014, 344, 65-69.

40. Woo, S.; Rothemund, P. W., Programmable Molecular Recognition Based on the Geometry of DNA Nanostructures. Nat. Chem. 2011, 3, 620-627.

41. Kilchherr, F.; Wachauf, C.; Pelz, B.; Rief, M.; Zacharias, M.; Dietz, H., Single-Molecule Dissection of Stacking Forces in DNA. Science 2016, 353, aaf5508.

42. Jungmann, R.; Scheible, M.; Kuzyk, A.; Pardatscher, G.; Castro, C. E.; Simmel, F. C., DNA Origami-Based Nanoribbons: Assembly, Length Distribution, and Twist. Nanotechnology 2011, 22 .

43. Tigges, T.; Heuser, T.; Tiwari, R.; Walther, A., 3D DNA Origami Cuboids as Monodisperse Patchy Nanoparticles for Switchable Hierarchical Self-Assembly. Nano Lett. 2016, 16, 7870-7874. 
44. Li, Z.; Liu, M.; Wang, L.; Nangreave, J.; Yan, H.; Liu, Y., Molecular Behavior of DNA Origami in Higher-Order Self-Assembly. J. Am. Chem. Soc. 2010, 132, 13545-13552.

45. Liu, W.; Halverson, J.; Tian, Y.; Tkachenko, A. V.; Gang, O., Self-Organized Architectures from Assorted DNA-Framed Nanoparticles. Nat. Chem. 2016, 8, 867.

46. Wang, P.; Gaitanaros, S.; Lee, S.; Bathe, M.; Shih, W. M.; Ke, Y., Programming SelfAssembly of DNA Origami Honeycomb Two-Dimensional Lattices and Plasmonic Metamaterials. J. Am. Chem. Soc. 2016, 138, 7733-7740.

47. Liu, W.; Zhong, H.; Wang, R.; Seeman, N. C., Crystalline Two-Dimensional DNAOrigami Arrays. Angew. Chem. 2011, 123, 278-281.

48. Zhang, T.; Hartl, C.; Frank, K.; Heuer-Jungemann, A.; Fischer, S.; Nickels, P. C.; Nickel, B.; Liedl, T., 3D DNA Origami Crystals. Adv. Mater. 2018, 30, 1800273.

49. Endo, M.; Sugita, T.; Katsuda, Y.; Hidaka, K.; Sugiyama, H., Programmed-Assembly System Using DNA Jigsaw Pieces. Chem. Eur. J. 2010, 16, 5362-5368.

50. Rajendran, A.; Endo, M.; Katsuda, Y.; Hidaka, K.; Sugiyama, H., Programmed TwoDimensional Self-Assembly of Multiple DNA Origami Jigsaw Pieces. ACS Nano 2010, 5, 665671.

51. Ke, Y.; Ong, L. L.; Shih, W. M.; Yin, P., Three-Dimensional Structures Self-Assembled from DNA Bricks. Science 2012, 338, 1177-1183.

52. Sun, W.; Boulais, E.; Hakobyan, Y.; Wang, W. L.; Guan, A.; Bathe, M.; Yin, P., Casting Inorganic Structures with DNA Molds. Science 2014, 346, 1258361.

53. Zhao, Z.; Liu, Y.; Yan, H., Organizing DNA Origami Tiles into Larger Structures Using Preformed Scaffold Frames. Nano Lett. 2011, 11, 2997-3002.

54. Schulman, R.; Winfree, E., Synthesis of Crystals with a Programmable Kinetic Barrier to Nucleation. Proc. Natl. Acad. Sci. USA 2007, 104, 15236-15241.

55. Mohammed, A. M.; Schulman, R., Directing Self-Assembly of DNA Nanotubes Using Programmable Seeds. Nano Lett. 2013, 13, 4006-4013.

56. Agrawal, D. K.; Jiang, R.; Reinhart, S.; Mohammed, A. M.; Jorgenson, T. D.; Schulman, R., Terminating DNA Tile Assembly with Nanostructured Caps. ACS Nano 2017, 11, 97709779.

57. Greschner, A. A.; Bujold, K. E.; Sleiman, H. F., Controlled Growth of DNA Structures from Repeating Units Using the Vernier Mechanism. Biomacromolecules 2014, 15, 3002-3008. 
58. Li, X.; Hao, C.; Tian, C.; Wang, P.; Mao, C., Vernier Assembly: Controlling DNA Polymerization Via Length Mismatching. Chem. Commun. 2014, 50, 6361-6363.

59. Hagan, M. F.; Grason, G. M., Equilibrium Mechanisms of Self-Limiting Assembly. 2020, arXiv:2007.01927. arXiv. https://arxiv.org/abs/2007.01927 (accessed November 16, 2020.

60. Cervantes-Salguero, K.; Hamada, S.; Nomura, S.-i. M.; Murata, S., Polymorphic RingShaped Molecular Clusters Made of Shape-Variable Building Blocks. Nanomaterials 2015, 5, 208-217.

61. Lee, L. K.; Ginsburg, M. A.; Crovace, C.; Donohoe, M.; Stock, D., Structure of the Torque Ring of the Flagellar Motor and the Molecular Basis for Rotational Switching. Nature 2010, 466, 996-1000.

62. Baker, M. A.; Hynson, R. M.; Ganuelas, L. A.; Mohammadi, N. S.; Liew, C. W.; Rey, A. A.; Duff, A. P.; Whitten, A. E.; Jeffries, C. M.; Delalez, N. J.; Morimoto, Y. V.; Stock, D.; Armitage, J. P.; Turberfield, A. J.; Namba, K.; Berry, R. M.; Lee, L. K., Domain-Swap Polymerization Drives the Self-Assembly of the Bacterial Flagellar Motor. Nat. Struct. Mol. Biol. 2016.

63. Katen, S.; Zlotnick, A., The Thermodynamics of Virus Capsid Assembly. Methods Enzymol. 2009, 455, 395-417.

64. Israelachvili, J. N.; Mitchell, D. J.; Ninham, B. W., Theory of Self-Assembly of Hydrocarbon Amphiphiles into Micelles and Bilayers. J. Chem. Soc., Faraday Trans. 2 1976, 72, 1525-1568.

65. Uchida, T.; Abe, K.; Endo, Y.; Ichiseki, S.; Akita, S.; Liu, S.; Aradachi, S.; Saito, M.; Fukuchi, A.; Kikkawa, T.; Dammaretz, T.; Kawamata, I.; Suzuki, Y.; Nomura, S.-i. M.; Murata, S., Revolving Vernier Mechanism Controls Size of Linear Homomultimer. Small 2017, 13, 1702158.

66. Kellenberger, E., Assembly in Biological Systems. In Ciba Foundation Symposium 7 Polymerization in Biological Systems; Wolstenholme, G. E. W. ; O'Connor, M.; Amsterdam, 1972; Vol. 7, pp 189-206.

67. Wagenknecht, T.; Bloomfield, V. A., Equilibrium Mechanisms of Length Regulation in Linear Protein Aggregates. Biopolymers 1975, 14, 2297-2309. 
68. Higuchi, H.; Funatsu, T.; Ishijima, A.; Okamura, N.; Ishiwata, S. i., Accumulated Strain Mechanism for Length Determination of Thick Filaments in Skeletal Muscle. I. Experimental Bases. J. Muscle Res. Cell Motil. 1986, 7, 491-500.

69. Alberts, B.; Bray, D.; Lewis, J.; Raff, M.; Roberts, K.; Watson, J. D., Molecular Biology of the Cell. 3rd ed.; Garland Science: New York, 1994.

70. Grason, G. M.; Bruinsma, R. F., Chirality and Equilibrium Biopolymer Bundles. Phys. Rev. Lett. 2007, 99, 098101.

71. Hough, L.; Jung, H.-T.; Krüerke, D.; Heberling, M.; Nakata, M.; Jones, C.; Chen, D.; Link, D. R.; Zasadzinski, J.; Heppke, G., Helical Nanofilament Phases. Science 2009, 325, 456460.

72. Gibaud, T.; Barry, E.; Zakhary, M. J.; Henglin, M.; Ward, A.; Yang, Y.; Berciu, C.; Oldenbourg, R.; Hagan, M. F.; Nicastro, D., Reconfigurable Self-Assembly through Chiral Control of Interfacial Tension. Nature 2012, 481, 348-351.

73. Li, S.; Roy, P.; Travesset, A.; Zandi, R., Why Large Icosahedral Viruses Need Scaffolding Proteins. Proc. Natl. Acad. Sci. USA 2018, 115, 10971-10976.

74. Meng, G.; Paulose, J.; Nelson, D. R.; Manoharan, V. N., Elastic Instability of a Crystal Growing on a Curved Surface. Science 2014, 343, 634-637.

75. Grason, G. M., Perspective: Geometrically Frustrated Assemblies. J. Chem. Phys. 2016, 145, 110901.

76. Penrose, L. S., Self-Reproducing Machines. Sci. Am. 1959, 200, 105-117.

77. Snodin, B.; Randisi, F.; Mosayebi, M.; Šulc, P.; Schreck, J.; Romano, F.; Ouldridge, T.; Tsukanov, R.; Nir, E.; Louis, A., Introducing Improved Structural Properties and Salt Dependence into a Coarse-Grained Model of DNA. J. Chem. Phys. 2015, 142, 234901.

78. Ouldridge, T. E.; Hoare, R. L.; Louis, A. A.; Doye, J. P.; Bath, J.; Turberfield, A. J., Optimizing DNA Nanotechnology through Coarse-Grained Modeling: A Two-Footed DNA Walker. ACS Nano 2013, 7, 2479-2490.

79. Schreck, J. S.; Romano, F.; Zimmer, M. H.; Louis, A. A.; Doye, J. P., Characterizing DNA Star-Tile-Based Nanostructures Using a Coarse-Grained Model. ACS Nano 2016, 10, $4236-4247$. 
80. Hong, F.; Jiang, S.; Lan, X.; Narayanan, R. P.; Šulc, P.; Zhang, F.; Liu, Y.; Yan, H., Layered-Crossover Tiles with Precisely Tunable Angles for 2D and 3D DNA Crystal Engineering. J. Am. Chem. Soc. 2018, 140, 14670-14676.

81. Berengut, J. F.; Berengut, J. C.; Doye, J. P.; Prešern, D.; Kawamoto, A.; Ruan, J.;

Wainwright, M. J.; Lee, L. K., Design and Synthesis of Pleated DNA Origami Nanotubes with Adjustable Diameters. Nucleic Acids Res. 2019, 47, 11963-11975.

82. Ouldridge, T. E.; Šulc, P.; Romano, F.; Doye, J. P.; Louis, A. A., DNA Hybridization Kinetics: Zippering, Internal Displacement and Sequence Dependence. Nucleic Acids Res. 2013, $41,8886-8895$.

83. Ouldridge, T. E.; Louis, A. A.; Doye, J. P., Structural, Mechanical, and Thermodynamic Properties of a Coarse-Grained DNA Model. J. Chem. Phys. 2011, 134, $02 B 627$.

84. Snodin, B. E.; Schreck, J. S.; Romano, F.; Louis, A. A.; Doye, J. P., Coarse-Grained Modelling of the Structural Properties of DNA Origami. Nucleic Acids Res. 2019, 47, 15851597.

85. Shi, Z.; Castro, C. E.; Arya, G., Conformational Dynamics of Mechanically Compliant DNA Nanostructures from Coarse-Grained Molecular Dynamics Simulations. ACS Nano 2017, $11,4617-4630$.

86. Sharma, R.; Schreck, J. S.; Romano, F.; Louis, A. A.; Doye, J. P., Characterizing the Motion of Jointed DNA Nanostructures Using a Coarse-Grained Model. ACS Nano 2017, 11, 12426-12435.

87. Engel, M. C.; Smith, D. M.; Jobst, M. A.; Sajfutdinow, M.; Liedl, T.; Romano, F.; Rovigatti, L.; Louis, A. A.; Doye, J. P., Force-Induced Unravelling of DNA Origami. ACS Nano 2018, 12, 6734-6747.

88. Kumar, S.; Rosenberg, J. M.; Bouzida, D.; Swendsen, R. H.; Kollman, P. A., The Weighted Histogram Analysis Method for Free-Energy Calculations on Biomolecules. I. The Method. J. Comput. Chem. 1992, 13, 1011-1021.

89. de Gennes, P.-G., Films of Polymer-Solutions. Comptes Rendus De L Academie Des Sciences Serie II 1985, 300, 839-843.

90. Ruckenstein, E.; Li, B., Steric Interactions between Two Grafted Polymer Brushes. J. Chem. Phys. 1997, 107, 932-942. 
91. Lenz, M.; Witten, T. A., Geometrical Frustration Yields Fibre Formation in SelfAssembly. Nat. Phys. 2017, 13, 1100-1104.

92. Douglas, S. M.; Marblestone, A. H.; Teerapittayanon, S.; Vazquez, A.; Church, G. M.;

Shih, W. M., Rapid Prototyping of 3D DNA-Origami Shapes with Cadnano. Nucleic Acids Res. 2009, 37, 5001-5006.

93. Kim, D.-N.; Kilchherr, F.; Dietz, H.; Bathe, M., Quantitative Prediction of 3D Solution Shape and Flexibility of Nucleic Acid Nanostructures. Nucleic Acids Res. 2011, 40, 2862-2868. 94. Schneider, C. A.; Rasband, W. S.; Eliceiri, K. W., NIH Image to Imagej: 25 Years of Image Analysis. Nat. Methods 2012, 9, 671.

For Table of Contents Only

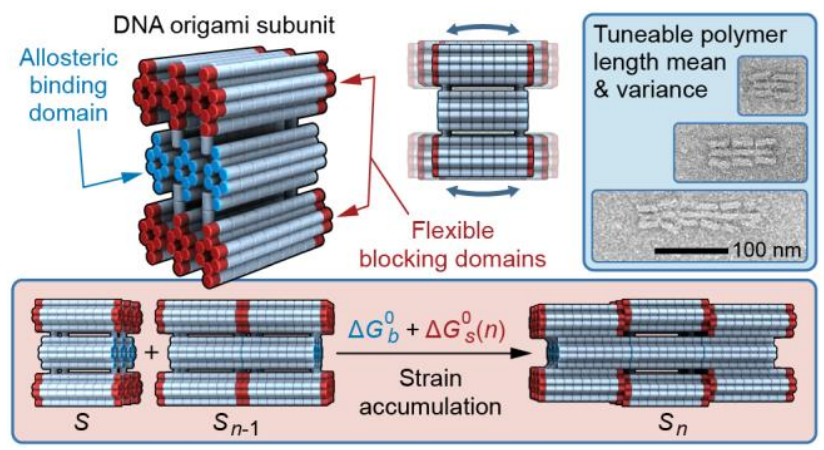

